\title{
Caracterização dos confinamentos de bovinos no Estado do Pará
}

\author{
Characterization of cattle feedlots in the Pará State \\ Caracterización de los confinamientos de ganado en el Estado de Pará
}

Recebido: 10/04/2021 | Revisado: 16/04/2021 | Aceito: 25/04/2021 | Publicado: 09/05/2021

Débora Alves de França Freire ORCID: https://orcid.org/0000-0003-0000-1508 Universidade Federal Rural da Amazônia, Brasil E-mail: debsfr20@gmail.com

Bruno Cabral Soares ORCID: https://orcid.org/0000-0003-1803-8907 Universidade Federal Rural da Amazônia, Brasil E-mail: brunocs@ veterinario.med.br

Aníbal Coutinho do Rêgo ORCID: https://orcid.org/0000-0002-5452-0832 Universidade Federal Rural da Amazônia, Brasil E-mail: anibalcr@gmail.com

Bruno Moura Monteiro ORCID: https://orcid.org/0000-0003-4272-9740 Universidade Federal Rural da Amazônia, Brasil

E-mail: brunomouramonteiro@hotmail.com

Letícia de Abreu Faria

ORCID: https://orcid.org/0000-0002-2506-2246 Universidade Federal Rural da Amazônia, Brasil

E-mail: leticia.faria@ufra.edu.br

Arlison Santos dos Santos

ORCID: https://orcid.org/0000-0001-7720-0121 Universidade Federal Rural da Amazônia, Brasil

E-mail: arlisonsantossmg2016@gmail.com

Andreza Araújo Cardoso

ORCID: https://orcid.org/0000-0001-9995-6974 Universidade Federal Rural da Amazônia, Brasil E-mail: andrezaaraujo937@gmail.com

Núbia de Fátima Alves dos Santos ORCID: https://orcid.org/0000-0001-7725-1412 Universidade Federal Rural da Amazônia, Brasil E-mail: nubia.santos@ufra.edu.br

Marcos Antônio Souza dos Santos ORCID: https://orcid.org/0000-0003-1028-1515 Universidade Federal Rural da Amazônia, Brasil E-mail: marcos.marituba@gmail.com

\begin{abstract}
Resumo
O artigo caracteriza os sistemas de confinamento de bovinos nas microrregiões de Cametá, Guamá, Tomé-Açu, Bragantina e Paragominas, estado do Pará. Foram entrevistados 20 produtores com o uso de questionário semiestruturado, contendo perguntas abertas e fechadas. A seleção da amostra apoiou-se na técnica bola de neve e as entrevistas foram realizadas em agosto e setembro de 2019. O roteiro da entrevista continha 101 perguntas direcionadas a identificação do proprietário, caracterização geral da propriedade e caracterização da unidade de produção, considerando os animais e a estrutura do sistema de produção. Os dados foram armazenados em planilha eletrônica do Microsoft Excel® e analisados no IBM SPSS Statistics ${ }^{\circledR}$ versão 22.0. A expansão do sistema de confinamento na região é recente e tem crescido nos últimos 10 anos. Os produtores possuem médio a alto nível de escolaridade, o que facilita ao acesso à tecnologia e assistência técnica que foi observado em todos os casos. Na maioria das propriedades a atividade é realizada na época seca para mantença de ganho de peso como principal objetivo. Modo geral, a estrutura é adequada para o processo produtivo, com genética melhorada nos rebanhos e poucos casos de mortalidades por doenças na fase terminal do confinamento. Programas de qualidade de carne nos frigoríficos podem incentivar ao aumento da adoção desse sistema de produção na pecuária bovina paraense.
\end{abstract}

Palavras-chave: Bovinocultura de corte; Sistemas de produção, Intensificação produtiva; Amazônia Brasileira.

\section{Abstract}

The objective of this work was to characterize the containment systems in the microregions of Cametá, Guamá, Tomé-Açu, Bragantina and Paragominas in the state of Pará. For this, 20 producers were interviewed using a semistructured script containing open and closed questions by qualitative and quantitative approaches. The selection of the 
sample was based on the snowball technique and the application of the information was evaluated in August and September 2019. The interview script contained 101 questions aimed at identifying the owner, general characterization of the property, characterization of the production unit considering animals and the structure of the production system. The data obtained were obtained in Excel spreadsheets and processed with IBM SPSS Statistics software version 22.0SPSS to perform descriptive statistical analyzes and inferences compared with the literature. We conclude that the exploitation of the containment system in the region is recent and has grown over the past 10 years. The producers have a medium to high level of education, which facilitates access to technology and technical assistance that was observed in all cases. In most properties the activity is carried out in the dry season to maintain weight gain as the main objective. In general, there is an adequate structure to carry out activities, with the inclusion of improved genetics in herds and few cases of disease mortality in the terminal phase of beef cattle. Meat quality programs in slaughterhouses can encourage an increase in the use of technology in the region.

Keywords: Beef cattle; Production systems, Productive intensification; Brazilian Amazon.

\section{Resumen}

El objetivo de este trabajo fue caracterizar los sistemas de confinamiento en las microrregiones de Cametá, Guamá, Tomé-Açu, Bragantina y Paragominas en el estado de Pará. Para ello, se entrevistó a 20 productores utilizando un guión semiestructurado que contenía abierto y cerrado preguntas mediante enfoques cualitativos y cuantitativos. La selección de la muestra se basó en la técnica de bola de nieve y se evaluó la aplicación de la información en agosto y septiembre de 2019. El guión de la entrevista contenía 101 preguntas dirigidas a identificar al dueño, caracterización general de la propiedad, caracterización de la unidad de producción considerando animales y la estructura del sistema de producción. Los datos obtenidos se obtuvieron en hojas de cálculo de Excel y se procesaron con el software IBM SPSS Statistics versión 22.0SPSS para realizar análisis estadísticos descriptivos e inferencias comparadas con la literatura. Concluimos que la explotación del sistema de contención en la región es reciente y ha crecido en los últimos 10 años. Los productores tienen un nivel de educación medio a alto, lo que facilita el acceso a la tecnología y asistencia técnica que se observó en todos los casos. En la mayoría de las propiedades la actividad se realiza en época seca para mantener la ganancia de peso como principal objetivo. En general, existe una estructura adecuada para realizar las actividades, con inclusión de genética mejorada en los rebaños y pocos casos de mortalidad por enfermedades en la fase terminal del ganado de carne. Los programas de calidad de la carne en los mataderos pueden fomentar un aumento en el uso de tecnología en la región.

Palabras clave: Ganado vacuno; Sistemas de producción, Intensificación productiva; Amazonia Brasileña.

\section{Introdução}

A pecuária brasileira é importante nos âmbitos nacional e mundial. É destaque em rankings mundiais como maior rebanho (221,81 milhões), maior exportador de bovinos (20\% da sua produção total) e segundo maior produtor de carne, contribuindo com $31 \%$ do PIB do agronegócio brasileiro. Os sistemas de terminação a pasto são os maiores contribuintes na produção total (87\%), isto ocorre principalmente por dois fatores: o clima tropical e a vasta extensão territorial. Desta man eira, apenas $13 \%$ do rebanho são mantidos em confinamentos, totalizando aproximadamente 4.046.217 de animais mantidos neste sistema (Abiec, 2018; Alves, Carvalho \& Silva, 2014). Use o parágrafo como modelo.

O confinamento é uma estratégia que tem sido empregada por muitos pecuaristas ao longo dos anos em pelo menos uma fase do sistema de produção. Nas últimas quatro décadas o rebanho nacional cresceu substancialmente e a atividade migrou para outras regiões do país, sobretudo, para o Norte do Brasil. Tanto o avanço da agricultura, como as maiores demandas por carnes exigiram maior intensificação produtiva na bovinocultura de corte. A adoção de sistemas de produção como, por exemplo, o confinamento, é um dos fatores responsáveis pelo aumento de produtividade nos sistemas de produção (Gomes, Feijó, \& Chiari, 2017).

O confinamento de bovinos de corte permite a alocação de um grande número de animais por área. Tal estratégia pode auxiliar na redução do ciclo produtivo, encurtamento de fases do sistema de produção e maior competitividade da atividade. Isso é possibilitado principalmente por meio de dietas que permitam expressar o máximo do potencial genético dos animais, que reduz a idade ao abate e proporciona melhores acabamentos (Lanna \& Almeida, 2005). Diferentes objetivos e disponibilidades de recursos são fatores determinantes para que os sistemas de confinamento brasileiros sejam diversificados, possibilitando inúmeras combinações entre tipos de instalações, raças e dietas (Thiago, 1996).

A bovinocultura do estado do Pará é significativa para pecuária nacional, visto que detém o quinto maior rebanho 
bovino brasileiro, com estimativa de 20.475 .541 cabeças. O efetivo do estado tem participação de $9,23 \%$ no rebanho total do Brasil, e 89,56\% dos seus animais são destinados exclusivamente para corte (Abiec, 2018). Os bovinos terminados no Pará são, na maioria, terminados a pasto, e a grande extensão territorial é um fator determinante para a utilização deste método de terminação. Porém, dados de desmatamentos na região têm sido associados com a pecuária desenvolvida na Amazônia, e empresas envolvidas ou favorecidas por este setor são pressionadas constantemente a se desassociarem da atividade pecuária.

Nesse contexto, a adoção de estratégias de manejo que aumentem a produtividade e diminuam a quantidade de área utilizada, como é o caso dos confinamentos, estão sendo empregadas por muitos produtores (Wilkison, 2015). A escassez hídrica enfrentada em parte do estado do Pará, por aproximadamente 4 a 6 meses do ano, e o crescimento da produção de soja e milho, são outros fatores determinantes para o emprego desta tecnologia (Bastos et al., 2005; Maia, 2014). Em função disso tem ocorrido a expansão de confinamentos de bovinos, nos últimos dez anos, no estado do Pará (Vale et al. 2019).

A importância do presente trabalho se justifica pela carência de diagnósticos que caracterizem e interpretem a funcionalidade dos sistemas de confinamento no estado do Pará, principalmente, nas microrregiões com maior pressão sobre o uso da terra, como aquelas mais próximas da Região Metropolitana de Belém. Dessa forma faz-se necessário o levantamento dessas informações, que irão contribuir para o conhecimento de todos os atores da cadeia pecuária (produtores, frigoríficos e instituições) e com políticas públicas de incentivo ao agronegócio paraense. Com base no exposto, o objetivo do trabalho foi caracterizar os sistemas de confinamento nas microrregiões de Cametá, Guamá, Tomé-Açu, Bragantina e Paragominas, estado do Pará.

\section{Metodologia}

O estudo foi realizado nas microrregiões Cametá, Guamá, Tomé-Açu, Bragantina e Paragominas, localizadas nas mesorregiões nordeste e sudeste do estado do Pará. Os territórios dessas mesorregiões são de $297.000 \mathrm{~km}^{2}$ e $83.553,5 \mathrm{~km}^{2}$, respectivamente, com solo de textura variando de média a muito argilosa, classificado como Argissolo (IBGE, 1990).

O clima predominante nas microrregiões é do tipo Aw da classificação de Köpen (clima tropical chuvoso, com expressivo período de estiagem) e Am (clima tropical chuvoso, com curto período de estiagem) (Bastos et al., 2005). Ambas as regiões possuem precipitação entre $750 \mathrm{~mm}$ e $1800 \mathrm{~mm}$, umidade de $80 \%$ e temperaturas médias anuais próxima a superiores a $18^{\circ} \mathrm{C}$.

O trabalho caracteriza-se por pesquisa de campo realizada com produtores e/ou técnicos responsáveis pelos confinamentos. No total foram 20 entrevistados, em que o número de entrevistados é diretamente correlacionado com a existência de sistema de confinamento na propriedade. As entrevistas foram realizadas por meio de um roteiro semiestruturado. Este tipo de entrevista é definido pela combinação de perguntas abertas e fechadas, em que o informante tem a possibilidade de discorrer sobre o tema proposto (Boni \& Quaresma, 2005). Na coleta de dados optou-se por combinar abordagens qualitativas e quantitativas, que para Gerhardt e Silveira (2009) são estratégias complementares.

A seleção da amostra apoiou-se na técnica bola de neve. Nesta técnica cada entrevistado indica outras propriedades relevantes para a pesquisa, pois pressupõe que há uma ligação entre os membros da população dado pela característica de interesse (Faugier \& Sargeant, 1997). As primeiras propriedades foram selecionadas por meio de informações obtidas de diferentes fontes selecionadas, como por exemplo, auxílio de professores e técnicos da Universidade Federal Rural da Amazônia (UFRA) que possuem contato ou conhecimento do público alvo. Após, as próximas seleções para aplicação da entrevista foram por meio de indicações dos entrevistados primários.

As entrevistas foram realizadas nos meses de agosto e setembro de 2019. O roteiro de entrevista abordou sobre identificação do proprietário, caracterização geral da propriedade e caracterização da unidade de produção que envolve informações sobre os animais de confinamento, da estrutura do confinamento e do sistema de produção. Os dados obtidos 
foram armazenados em planilhas eletrônicas do Microsoft Excel@ e analisados no IBM SPSS Statistics® versão 22.0.

\section{Resultados e Discussão}

\subsection{Características dos produtores}

A adoção de sistemas de confinamento nas microrregiões em estudo é recente. A maioria dos confinamentos teve início nos últimos três anos (45,0\%), e os demais entre 3,1 a 6 anos (25,0\%) e entre 6,1 a 9 anos (30,0\%). Estes resultados indicam que o sistema de confinamento tem crescido nos últimos 10 anos e ainda está em desenvolvimento.

A maioria dos proprietários $(61,1 \%)$ não reside na propriedade. A idade média deles é de 52 anos. Quanto ao nível de escolaridade dos produtores, $40 \%$ possuem ensino superior completo, no entanto, a formação da maioria não é relacionada às ciências agrárias. Este nível de escolaridade pode facilitar a adoção de medidas estratégicas e técnicas de manejo fundamentais para o desenvolvimento da atividade (Bezerra et al., 2013).

Os dois principais motivos que levaram os produtores ao emprego do sistema de confinamento em suas propriedades são: manter o bom desempenho dos animais na época seca e acelerar o ganho de peso dos animais. Diferentemente do que se aplica a nível nacional, que está relacionado ao aumento da produtividade, nas propriedades analisadas, a mantença de ganho de peso ainda é a principal motivação para o emprego desta tecnologia (Lanna \& Almeida, 2005).

Constatou-se que $65 \%$ dos produtores nunca receberam financiamento voltado para atividade de confinamento, pois possuíam recurso próprio para o funcionamento da atividade. Os 35\% dos produtores que solicitaram o benefício destinaram estes recursos, principalmente, para maquinários e/ou equipamentos.

\subsection{Características das unidades de produção}

Os confinamentos cobertos pela pesquisa estão distribuídos em 14 municípios, com maior concentração no município de Paragominas (25\%), Ipixuna do Pará (15\%) e Tomé-açu (15\%). A microrregião de Paragominas configura o maior polo agrícola do estado, onde a principal atividade é a pecuária, seguida da agricultura de grande porte (Alves, Carvalho \& Silva, 2014). Este fato contribuiu significativamente a relevância apresentada nos dados para os municípios dessa microrregião.

As propriedades pesquisadas estão em tamanho de área concentradas nas faixas de até 5.000 ha (45\%), de 5.001 a 10.000 ha (15\%) e maiores de 10.000 ha (40\%), onde todas possuem reserva legal estando de acordo com a Lei 12.651/2012, em que todo imóvel rural deve manter uma área com cobertura de vegetação nativa. Estes apresentam no mínimo 50\% da sua reserva legal (Brasil, 2012).

Em todas as propriedades existem fontes de água, entre essas as mais citadas foram poços (85\%), represas (50\%), rios (35\%), igarapés (25\%) e nascentes (15\%), e a maioria dos produtores (60\%) mantem vegetação para proteção da fonte de água. Diferentes dados foram observados por Soares (2017), para as propriedades leiteiras localizadas no município de Rondon, situado em uma das microrregiões em estudo, as fontes de água mais frequentes foram represas $(65,5 \%)$ e rios $(56,4 \%)$.

A maior parcela dos produtores (50\%) trabalha somente com pecuária de corte, outros (45\%) além da pecuária de corte, têm a agricultura como atividade secundária. No entanto, em 85,7\% dos confinamentos, a produção vegetal se faz presente com o objetivo da alimentação dos animais em confinamento ou semiconfinamento.

Com relação ao relevo, é predominante nas propriedades levantadas a baixa declividade do terreno, 50\% dos entrevistados responderam que as propriedades eram pouco declivosas (declividade menos do que 8\%). Em seguida $44,4 \%$ afirmaram que o relevo era suavemente ondulado (com 9-20\% de declividades) e os demais responderam que a declividade era acentuada. Diante disso, pode-se inferir que a declividade dos terrenos é adequada para a implantação dos sistemas de confinamento (Thiago, 1996) e que áreas com potencial para o desenvolvimento da agropecuária são destinadas a esse tipo de exploração. 
O acesso das vias principais às propriedades é considerado fácil, em 94,4\% dos casos. Destaca-se que esse é um aspecto importante na redução de perdas econômica dos produtores no frigorífico, pois está diretamente relacionado a perdas em decorrência do transporte. Andrade et al. (2008) constataram que o transporte de animais por vias não-pavimentadas é um dos fatores de maior proporção de lesões de carcaça. As perdas por lesões de carcaça decorrentes de transporte podem ultrapassar R\$ 200 mil/ano para os pecuaristas (Polizel Neto et al., 2015).

\subsection{Caracterização dos animais de confinamento}

O tamanho dos confinamentos foi estratificado em três níveis: (i) menos de 5 mil animais, (ii) de 5 a 10 mil animais e (iii) mais de 10 mil animais. Constatou-se que 50\% das propriedades estão concentradas no primeiro nível e 30\% no segundo nível. Mas 20\% já possuem mais de 10 mil animais confinados.

A composição racial dos rebanhos destinados ao sistema na maior parte é nelore e cruzamento industrial (55\%). Os cruzamentos mencionados mais comuns são Nelore x Aberdeen Angus e Nelore x Charolês, representando cerca de apenas $10 \%$ do total dos animais. No entanto, nas propriedades destinadas a carne Premium chega a 50\% do total de confinados. Esses dados diferem do observado por Carenagto et al. (2017) em confinamentos na região do meio oeste catarinense, em que o gado europeu (Angus e Hereford) são predominantes. Os resultados obtidos quanto aos cruzamentos na presente pesquisa podem ser um indicativo de que mesmo com poucos programas de qualidade de carne existentes no estado do Pará, os produtores têm buscado incluir nos planteis animais com genética que favorecem a produção de carne.

As categorias de maior prevalência nos confinamentos da região são machos inteiros e fêmeas jovens (55\%), estas representam cerca de $10 \%$ dos animais de confinamento e são destinadas para programas de qualidade de carne.

Os produtores das regiões estudadas são remunerados por rendimento de carcaça, que é um dos critérios de pagamentos dos frigoríficos brasileiros, por isso observou-se com maior predominância de boi gordo. Porém, Pires (2010) afirma que esta realidade no Brasil é diretamente causada pela proibição de anabolizantes na pecuária bovina brasileira segundo a instrução Normativa nº10, de 27 de abril de 2001 do MAPA (Ministérios de Agricultura, Pecuária e Abastecimento). Isso explica o menor uso de animais de outras categorias em confinamento pela expectativa de maiores taxas de ganho de peso, proporcionada por bovinos inteiros.

Em todas as propriedades avaliadas é realizada a apartação de animais por lote. Os critérios realizados pela maior parte dos produtores (40\%) são sexo, raça e peso. Este manejo é importante para evitar principalmente competitividade no cocho e, neste sentindo, garantir melhor bem-estar e desempenho aos animais (Quintiliano \& Costa, 2007).

O peso de entrada dos animais nos confinamentos está entre 350 a $450 \mathrm{~kg}$. Na saída para o abate, o peso pode variar entre 500 a $600 \mathrm{~kg}$, com rendimento de carcaça médio de 54\%. Deve-se ressaltar que aqueles que entram mais pesados no sistema, não necessariamente possuem peso de saída maior, desta forma, estes passam maior tempo sendo alimentados a pasto como forma de reduzir os dias no confinamento. A faixa etária média dos animais apresentada pelos entrevistados é de 22 meses e o ganho é de 1,5kg/dia/animal. Estas informações de produção corroboram com os dados encontrados em vários confinamentos no Brasil descritos por Oliveira (2017).

A compra de animais para a fase de terminação é realizada por $85 \%$ dos produtores, com preferência pelos animais de recria (40\%). O baixo valor desses animais na época da entressafra (média $\mathrm{R} \$ 5,43 / \mathrm{kg}$ ) é o principal critério de compra dos produtores (35\%), seguidos preço e da qualidade dos animais (25\%).

A produção deste sistema é destinada para frigoríficos (55\%) e/ou exportações (45\%), em que o rendimento de carcaça como o maior critério (55\%) exigido pelos compradores (frigorifico). Este fato reafirma a grande produção de boi gordo gerado no estado.

O custo da diária nas propriedades avaliadas está entre $\mathrm{R} \$ 1,00$ e 5,00 em 10\% dos casos e entre $\mathrm{R} \$ 5,10$ e 10,00 em 
$70 \%$ dos casos. No que se refere a comercialização da produção, 30\% dos entrevistados responderam que é referente ao preço da arroba do dia e os $50 \%$ afirmaram em média que vendem $\mathrm{R} \$ 4$ a $7 / \mathrm{kg}$ de peso vivo. Valores maiores que $\mathrm{R} \$ 10 \mathrm{~kg}$ de peso vivo foram observados para estabelecimentos de produção de animais Premium, pois os programas pagam 10\% a mais do valor da arroba do macho.

\subsection{Caracterização da infraestrutura do confinamento}

As estruturas de confinamentos, em 55,6\% propriedades, são menores do que 10 ha. Em média em cada estrutura de confinamento existem 80 animais/lote, que possuem capacidade estática no total entre 400 e 3.000 animais. Segundo Thiago (1996), o número máximo de animais/lote deve ser de 100 para facilitar o manejo. Observando que a maioria das propriedades estudadas apresentavam condições favoráveis para a execução da atividade

A época mais frequente em que se confinam animais na região é na época seca (85\%), e apenas três propriedades (15\%) confinam o ano todo. Dentre os que confinam apenas na época seca do ano, $45 \%$ responderam que são realizados dois giros. Entre os que confinam ao longo de todo o ano, um produtor executa três giros e outro quatro giros. De maneira geral a duração de cada giro é de três meses, em 95\% dos casos. Pires (2010) afirma que o confinamento de animais na época das águas apresenta muitas dificuldades, entre elas acúmulo de lamas nos currais, alta infestação de ectoparasitas, reduzida oferta de alimentos concentrados e a baixa competitividade com o boi criado a pasto, que nesta época tem custo reduzido. Portanto, confinar neste período se torna favorável somente se o produtor for favorecido por pagamento de prêmios, como foi observado em um dos casos nas propriedades em estudo.

Nas regiões de estudo, os confinamentos são parcialmente cobertos (55\%) e 45\% sem cobertura. Animais em sistemas parcialmente cobertos tendem apresentar melhor desempenho, principalmente em regiões tropicais, por proporcionarem ambientes mais confortáveis e favoráveis a expressão do desempenho (Mac-Lean et al., 2011). Em todas as propriedades o piso é do tipo chão batido, mas em 15\% delas há concreto ao redor dos comedouros/bebedouros como forma de evitar problemas sanitários aos animais.

A decisão quanto ao material utilizado para construção da estrutura de confinamento está relacionada aos recursos dos produtores e preferência. Observou-se entre os produtores a preferência por cercas do tipo arame liso, elétricas e madeira em $95 \%$ dos casos.

O tipo de cocho formato 'jota' é o mais utilizado pelos produtores (60\%). Tal utilização justifica-se pela estrutura que beneficia a redução de perdas ocasionadas pelos animais em alimentação. O material usado pelos produtores em $90 \%$ é o concreto, pois possui maior durabilidade comparado aos demais. Foi observado que em todas as fazendas avaliadas é usada estratégia para evitar competitividade dos animais no cocho. Normalmente é considerado o cálculo de capacidade levando-se em consideração a metragem linear de cocho, que segundo Thiago (1996), o espaço recomendável é de $70 \mathrm{~cm}$ linear por animal.

Em 50\% das propriedades a localização do bebedouro é próximo ao comedouro e compartilhado para dois currais. Em outras propriedades (35\%) o bebedouro é próximo ao fundo e compartilhado entre dois currais. Destaca-se que a divisão dos bebedouros pode reduzir o custo inicial de investimento na atividade, no entanto, capacidade e vazão devem ser analisadas para que atendam aos animais dos lotes. O concreto também é predominante como material utilizado dos bebedouros (85\%), e estes em 63,2\% dos casos têm capacidade de 400 a 2.000 litros.

Todos os produtores realizam o manejo de resíduos e $80 \%$ dos entrevistados afirmam que são retirados com auxílio de maquinários. Estima-se que $40 \%$ da matéria orgânica é eliminada nas fezes, com isso, os produtores possuem lago de decantação e outros destinam os resíduos para adubação de pasto. A retirada de dejetos e a destinação adequada reduzem ou eliminam multiplicação de moscas, presença de endo e ectoparasitas no rebanho e o impacto poluente do manejo inadequado 
ou descarte incorreto dos mesmos (Quadros, 2015).

\subsection{Caracterização do manejo alimentar}

Pires (2010) afirma que o nível de grãos na dieta é dependente de vários fatores econômicos, como, custos relativos da forragem, custos fixos, e dos preços relativos de venda dos animais. Em 70\% dos estabelecimentos ocorre a produção de grãos e volumosos, sendo comprados apenas os insumos complementares.

O milho é a cultura plantada em maior proporção nas propriedades (80\%), seguida do sorgo (40\%), capim (35\%) e cana-de-açúcar (5\%). Esses dados corroboram com a realidade nacional, em que, de modo geral, o milho é o mais utilizado nas elaborações de rações dos animais, e que, é comumente substituído por outros grãos de menores custos, em particular o sorgo (Pires, 2010). Dentre os métodos de utilização mais usuais dessa cultura na região, está a silagem e/ou fornecimento dele moído.

Em duas (10\%) propriedades não há fábricas de ração, que está relacionado a sua pequena capacidade de animais no confinamento. Parte dos estabelecimentos possuem estruturas com espaço de armazenamento suficiente para mais que 6.000 sacas e os outros com capacidade inferior, porém que atendem a sua demanda.

Há maquinários em todas as unidades produtivas destinadas para as atividades do confinamento. A quantidade observada em maior parte (80\%) é de 5 ou menos maquinários. Dentre esses estão, tratores, vagão misturador e pá carregadeira são os maquinários mais comuns nas propriedades.

A adaptação à dieta é realizada por $95 \%$ dos produtores em método mais de escala, que consiste na inclusão gradativa do concentrado na alimentação dos animais. Independente da proporção de concentrado adotada, se faz necessário a transição gradual de volumosos para concentrados para evitar diversos distúrbios metabólicos que podem ter efeitos no desempenho e característica de carcaça (Pires, 2010).

As dietas são compostas basicamente por silagem de milho ou milho moído, farelo de soja, capim e em algumas propriedades é utilizado o caroço de algodão e bagaço de cana, além destes, incluem-se também o núcleo e em alguns casos aditivos. O bagaço de cana utilizado em uma propriedade é oriundo do aproveitamento da indústria de sucroalcooleira, estratégia utilizada pelo produtor para diminuir os custos com a alimentação dos animais, visto que o confinamento e a indústria são do mesmo proprietário. O manejo de quatro alimentações/dia é o mais comum (65\%) nas regiões em estudo, com fornecimento em média $8 \mathrm{~kg} / \mathrm{dia} / \mathrm{animal}$. O cálculo da quantidade é feito na entrada pelo peso do animal e após o animal ser confinado é feita a leitura de cocho diária.

Todos os produtores relataram que recebem assistência técnica, com frequência variada entre diariamente (5,3\%), uma vez na semana (26,3\%), quinzenal durante o período de confinamento (15,8\%), uma vez ao mês durante o confinamento $(15,8 \%)$ e quando solicitam (31,6\%). Entre os profissionais que prestam assistência destacam-se os zootecnistas $(52,6 \%)$, seguido por agrônomos $(21,1 \%)$, médicos veterinários (10,5\%) e administradores $(15,8 \%)$.

\subsection{Caracterização do manejo sanitário}

Com relação ao manejo sanitário das propriedades, as vacinas anuais e preventivas (febre aftosa, raiva, vermífugos, carrapaticida) são realizadas antes da entrada no confinamento, e se necessário aplica-se as contra ecto e endoparasitas após. A maioria realiza vacina, $77,8 \%$ para doença respiratória bovina (BRD) como forma preventiva, mas que não observaram casos da doença. De modo geral, todos relataram serem raros os casos de problemas sanitários graves em animais confinados. No entanto, um dos entrevistados relatou que os casos de mortalidades observados decorrem de acidentes dos animais nas instalações ou brigas entre os mesmos. 


\section{Conclusão}

A exploração do sistema de confinamento nas microrregiões de Cametá, Guamá, Tomé-Açu, Bragantina e Paragominas no estado do Pará é recente e tem crescido nos últimos 10 anos, sobretudo nos três últimos anos.

Os produtores possuem médio a alto nível de escolaridade, o que facilita o acesso à tecnologia e assistência técnica que foi observado em todos os casos.

$\mathrm{Na}$ maioria das propriedades o confinamento é utilizado na época seca para mantença de ganho de peso como principal objetivo, exceto em propriedades em que a produção é destinada ao mercado específico de carne Premium.

De modo geral, há uma estrutura adequada para exercer as atividades, com inserção de animais derivados de cruzamentos que privilegiam a produção de carne e constataram-se também poucos casos de mortalidade por doenças na fase final do confinamento.

Programas de qualidade de carne nos frigoríficos podem incentivar ao aumento de sistemas de confinamento no estado do Pará.

\section{Referências}

Abiec (2018). Associação Brasileira Das Indústrias Exportadoras de Carnes. Perfil da pecuária no Brasil.

Alves, L. W. R., Carvalho, J. M., \& Silva, L. G. T. (2014). Diagnóstico agrícola do município de Paragominas, PA. Embrapa, 26 p.

Andrade, E. N. de. et al. (2008). Ocorrência de lesões em carcaças de bovinos de corte no Pantanal em função do transporte. Ciência Rural, 38 (7), 1192-1994.

Bastos, T. X. et al. (2005). Características agroclimáticas do munícipio de Paragominas. Embrapa, 21 p.

Bezerra, L. R. et al. (2013). Caracterização de propriedades agrícolas para pecuária de corte. Comunicata Scientiae. 4(1). 75-76.

Boni, V., \& Quaresma, S. J. (2005). Aprendendo a entrevistar: como fazer entrevistas em ciências sociais. Revista Eletrônica dos Pós-graduandos em sociologia Política da UFSC. 2(1) 70-76.

Brasil (2012). Lei $n^{\circ}$ 12.651, de 25 de maio de 2012. Dispõe sobre a proteção da vegetação nativa. Presidência da república.

Carenagto, J. P. et al. (2017). Caracterização dos confinamentos de bovinos de corte na região do meio oeste catarinense. Revista eletrônica Nutritime. 14(4) 6077-6078.

Faugier, J., \& Sargeant, M. (1997). Sampling hard to reach population. Journal of Advanced Nursing. 26(1). $790-797$.

Gerhardt, T. E., \& Silveira, D. F. (2009). Métodos de pesquisa.

Gomes, R. da C., Feijó, G. L. D., \& Chiari, L. (2017). Evolução e qualidade da pecuária brasileira.

Ibge (1990). Instituto brasileiro de geografia e estatística. Divisão regional do Brasil em mesorregiões e microrregiões geográficas. 137 p.

Lanna, D. P. D., \& Almeida, R. de (2005). A terminação de bovinos em confinamento. Visão agrícola. (3). 55-57.

Mac-Lean, P. A. B. et al. (2011). Sombra artificial e método de fornecimento de concentrado no comportamento e desempenho de bezerros desmamados. Acta Scientiarum Animal Sciences, 33(4). 2011.

Maia, J. T. da S. (2014). Análise bioeconômica da produção de novilhos precoces de diferentes grupos genéticos terminados em confinamento, em Paragominas, Pará. Dissertação (Mestrado em Ciência animal) - Universidade Federal do Pará, Pará.

Oliveira, F. S. de. (2017). Análise do sistema de confinamento de bovinos de corte no mercado brasileiro. Dissertação (Mestrado em agronegócio). Universidade de Brasília.

Pires, A. V. (2010). Bovinocultura de corte. 760 p.

Polizel Neto, A. et al. (2015). Perdas econômicas ocasionadas por lesões em carcaças de bovinos abatidos em matadouro-frigorífico do norte de Mato Grosso. Pesquisa Veterinária Brasileira, 35(4) 324-325.

Quadros, D.G. (2015). Sistema de produção de bovino de corte. In: Apostila técnica do curso sistema de produção de bovino de corte.

Quintiliano, M. H., \& Costa, M. J. R. P. da. (2019). Influência do bem-estar animal na eficiência de sistemas de produção intensivo de bovinos. <https://www1.ufmt.br/ufmt/unidade/userfiles/publicacoes/601b97e89c5b7262cc3b73bf66e2dd3a.pdf>.

Soares, B. C. (2017). Perfil das unidades produtoras e índices de desempenho produtivo da pecuária leiteira bovina de Rondon do Pará, estado do Pará. Tese Doutorado (Ciência animal) Instituto de Medicina Veterinária. Universidade Federal do Pará. Belém. 
Research, Society and Development, v. 10, n. 5, e35410514893, 2021

(CC BY 4.0) | ISSN 2525-3409 | DOI: http://dx.doi.org/10.33448/rsd-v10i5.14893

Thiago, L. R. L. de S. (1996). Confinamento de bovinos. Embrapa. 85 p.

Vale, B. P. et al. (2019). The Expansion of Intensive Beef Farming to the Brazilian Amazon. Global Environmental Change, 57 (2019) 101922

Wilkison, A. (2015). In Brazil, cattle industry begins to help fight deforestation. <https://www.sciencemag.org/news/2015/05/brazil-cattle-industry-beginshelp-fight-deforestation>. 